\title{
Círculos de estudios: redes de aprendizajes y fortalecimiento de capacidades pedagógicas
}

\author{
Mercy Karina Fuentes Rendón \\ p7002316078@ucvvirtual.edu.pe \\ https://orcid.org/0000-0002-8128-5137 \\ Universidad Cesar Vallejo, Piura - Perú \\ César Balladares Atoche \\ cesarballadares@hotmail.com \\ https://orcid.org/0000-0001-8284-7742 \\ Universidad Cesar Vallejo, Piura - Perú
}

\section{RESUMEN}

La educación actual, en cualquier parte del mundo, requiere de profesionales idóneos, con buena formación y capacidades donde prevalezca la excelencia y calidad. En este sentido el ministerio de educación del Ecuador desde el 2013, plantea el acompañamiento pedagógico y el rompimiento de paradigmas, para dar solución a problemas en los aprendizajes, a través de la formación continua, de manera presencial y en la actualidad de manera virtual. La línea o estrategia empleada, son los círculos de estudios destinados específicamente a educación inicial y preparatoria, en todo el territorio nacional. El objetivo general del presente es Determinar la incidencia de círculos de estudios redes de aprendizaje en el fortalecimiento pedagógico a docentes, distrito 12d03 Mocache Quevedo, Ecuador, 2021. Se utilizó una metodología de tipo básica diseño no experimental transaccional propositiva - explicativa, se contó con una población de 99 docentes y la muestra de 79 docentes educación inicial, los instrumentos para recabar información fueron la encuesta y cuestionarios. Los resultados evidencian que círculos de estudios, redes de aprendizaje su fortalecimiento pedagógico a los docentes se encuentra en un nivel bajo según los datos estadísticos analizados y es en base a la opinión de los encuestados y en relación a las dimensiones.

Palabras claves: fortalecimiento; aprendizaje; estrategia; educación 


\title{
Study circles: Learning and strengthening networks pedagogical skills
}

\begin{abstract}
Today's education, anywhere in the world, requires suitable professionals, with good training and skills where excellence and quality prevail. In this sense, the Ministry of Education of Ecuador since 2013, proposes pedagogical support and the breaking of paradigms, to solve problems in learning, through continuous training, in person and currently virtually. The line or strategy used is the study circles specifically intended for initial and preparatory education, throughout the national territory. The general objective of this present is to determine the incidence of study circles learning networks in the pedagogical strengthening of teachers, district 12d03 Mocache - Quevedo, Ecuador, 2021. A methodology of a basic type non-experimental transactional propositional design - explanatory was used, it was counted With a population of 99 teachers and a sample of 79 early childhood teachers, the instruments to collect information were the survey and questionnaires. The results show that study circles, learning networks, their pedagogical strengthening for teachers is at a low level according to the statistical data analyzed and is based on the opinion of the respondents and in relation to the dimensions.
\end{abstract}

.Key words: empowerment; learning; strategy; education.

Artículo recibido: 30 noviembre. 2021 Aceptado para publicación: 29 diciembre 2021 Correspondencia: p7002316078@ucvvirtual.edu.pe Conflictos de Interés: Ninguna que declarar 


\section{INTRODUCCION}

El acompañamiento pedagógico no es tarea fácil ya que los procesos que debe desplegase hacia los docentes implica, determinar en primera instancia el nivel de intervención, diseño de una planificación, organización de tiempos, recursos y materiales pertinentes, seguimiento y monitoreo para evidenciar los cambios ante la replanteamiento de la metodología y didáctica pedagógicos.

En mención se puede describir que el problema que da forma a esta investigación es la falta de aplicación de las orientaciones y fortalecimientos pedagógicos sugeridos en los círculos de estudios por parte de los docentes a la hora de desarrollar actividades y resolución de problemas además que no se ha podido evidencia o vestigio de monitoreo pedagógico ni sugerencias en referencia al recojo de información.

En cuanto a las capacitaciones que reciben mensualmente los docentes en la actualidad se han venido desarrollando a través de la modalidad virtual y la educación ha tenido que adaptarse a cambios superar dificultades y buscar nuevas formas de aprender, capacitación y formación, cabe recalcar que pese a los esfuerzos y resilencia mostrada por los docentes ante las circunstancias que envuelven a la población la educación ha decaído porque no se estuvo preparado para enfrentar situación a través del dominio de las tecnología en referencia a lo ante expuesto en un estudio realizado por la UNESCO, UNICEF, el Banco Mundial mencionan que solo un $81 \%$ de los profesionales de primaria han recibido capacitación y cumplen con sus funciones.

Gomero (2020). En su trabajo Plan de monitoreo integral pedagógico en el desempeño del docente de Educación Básica Regular. Ancash, 2019. Utilizo el método cuantitativo, con un estudio aplicado, de corte longitudinal, con un diseño experimental, de tipología pre experimental. La población de 1053 docentes de la UGEL Huaraz y Huarmey y la muestra por conveniencia fue de 127 docentes. Los instrumentos de recolección de datos la ficha de Monitoreo y Asistencia al Desempeño Docente.

Ministerio de educación del ecuador en julio 24 del (2020). Regula la educación abierta a nivel nacional a través del acuerdo MINEDUC 202000038-a. Este informe ministerial sugiere el uso de las herramientas virtuales en línea y la conectividad en tiempos de pandemia y distanciamiento social es lo más conveniente para la continuidad de la educación y propone el programa (homeshooling) educación en casa dirigida para todos 
los niveles educativos en cumplimiento del objetivo de asegurar el acceso y continuidad de la educación.

En cuanto a los resultados se determinó que el Monitoreo Integral Pedagógico influye significativamente en el desempeño de los docentes de zona urbana de Huaraz y zona rural de Huarmey con una media de 5.260 lo que implica encontrarse en el intervalo de $[5-8>$ puntos.

Previamente se ha desarrollado búsqueda de trabajos en relación al tema que da razón de ser al tema de este artículo en bibliotecas y repositorios de universidades locales, pero no se han encontrado trabajo aun realizados que permitan contrastar la información obtenida en la aplicación de instrumentos para el recojo de la información que da sustento y validez al presente trabajo de investigación.

El fortalecimiento pedagógico claramente es un recurso necesario para los docentes, y en los últimos años ha tornado indispensable en respuesta a los tiempos y adelantos en la educación, este tipo de fortaleciendo promueve que se reflexione la formación y generando una reestructuración en procesos y métodos de aprendizajes que se desarrollan en las aulas.

Conociendo esto se busca fundamentar a través de teorías los planteamientos antes mencionados y formular algunas acciones en lo que respecta al acompañamiento pedagógico específicamente en el área de educación inicial en el Ecuador, nos parece pertinente como primera medida, que es necesario determinar y reelaborar los fundamentos previamente considerados así también los procesos que están inmerso en el acompañamiento pedagógico en lo que respecta a la educación inicial siempre en búsqueda de la excelencia educativa.

La cualificación del talento humano que trabaja con la primera infancia se concibe como un proceso estructurado en el que las personas actualizan y amplían sus conocimientos, resinifican y movilizan sus creencias, imaginarios, concepciones y saberes, y fortalecen sus capacidades y prácticas cotidianas con el propósito de mejorar en un campo de acción determinado. (...) Esta se caracteriza por reconocer los saberes y experiencias en torno a la atención integral de la primera infancia de las y los participantes como punto de partida para la construcción de nuevos conocimientos, los cuales son el resultado de la reflexión que se realiza sobre las acciones cotidianas y la puesta en diálogo de los saberes y experiencias construidos en las prácticas con las comprensiones que la ciencia y la política 
tienen hoy del desarrollo de la primera infancia. Por esta razón, es clave tener en perspectiva que la cualificación del talento humano que trabaja con primera infancia se distancia de aquellos enfoques educativos que se centran de manera exclusiva en la transmisión de información, en la transferencia de metodologías o en la adquisición de miradas teóricas sobre temas referidos al desarrollo de las niñas y los niños. Pinto (2018, p.8).

Como segundo planteamiento se propone identificar cuáles son los vacíos, desafíos y fortalezas que presentan los docentes de educación inicial en referencia a la aplicación de las orientaciones recibidas como acompañamiento pedagógico en los círculos de estudios redes de aprendizajes con la finalidad de replantear o buscar las mejores herramientas que acorde a la realidad de cada contexto faciliten el desenvolvimiento y quehacer pedagógico.

En esta línea, la observación participante es el medio ideal para realizar descubrimientos, para examinar críticamente los conceptos teóricos y para anclarlos en realidades concretas, poniendo en comunicación distintas reflexividades. Veamos cómo los dos factores de la ecuación, observación y participación, pueden articularse exitosamente sin perder su productiva y creativa tensión. Guber (2001).

Posteriormente en vista de los diálogos sistemáticos y observaciones encontradas, tratadas, analizadas y expuesta en los resultados acertados sobre el acompañamiento pedagógico a los docentes de educación inicial en el Ecuador se propone una serie de actividades que promuevan el cumplimiento y pronta mejora de los procesos educativos a través del monitoreo en cuanto a que tan incidente y pertinentes resultan los fortalecimientos a la hora de desarrollar las clases por parte de los docentes y posteríos evaluación a los resultados recabados que involucran directamente las capacitaciones recibidas.

Se sustenta en la teoría humanista, La educación humanista, se aborda el tema de la motivación y la autorrealización educativa, ya que como seres humanos hay una tendencia a evolucionar hacia el pleno desarrollo integral y holístico. Fernández (2017). La presente teoría está relacionada estrechamente relacionada con las acciones que preceden al descubrimiento, exploración y el desarrollo de la creatividad de cada persona. 
La teoría Constructivista, El constructivismo es una teoría que sostiene que un individuo adquiere conocimientos y entiende las cosas mediante el contraste entre sus experiencias e ideas, es decir, el sujeto de aprendizaje se apropia del conocimiento siempre y cuando realice acciones que le permitan comparar situaciones nuevas con las que ya poseía. (Ordoñez et al., 2020).

Tiene que ver con la plasticidad y percepción de cada individuo y el desarrollo del pensamiento lógico específicamente tiene relación con la reflexión en cuanto a proceso de entrelace de las experiencias con las nuevas dando paso a mejorar el conocimiento, habilidades y destrezas.

\section{ESTRATEGIAS METODOLÓGICAS O MATERIALES Y MÉTODOS}

La metodología utilizada es de tipo básica diseño no experimental transaccional propositiva - explicativa, se contó con una población de 99 docentes y la muestra de 79 docentes educación inicial, los instrumentos con los que se recabaron la información fueron la encuesta y cuestionarios de la encuesta.

Cantillo (2018). Manifiesta que saber reflexionar sobre la propia práctica: ¿Es este el objetivo fundamental de la formación de los enseñantes por ende el acompañamiento pedagógico permitió que los docentes desarrollen una práctica reflexiva que transforma la práctica docente? Porque se utilizó un corte cualitativo que sigue el método de la investigación acción y búsqueda para determinar los resultados obtenidos y al analizarla proponer un plan de monitoreo y evaluación en las instituciones por parte de los círculos de estudios redes de aprendizaje.

\section{RESULTADOS Y DISCUSIÓN}

Al referirse a las variables círculos de estudios redes de aprendizaje y acompañamiento pedagógico procesada la información se puede evidenciar que

De acuerdo a la información recopilada se pude decir que las dos variables tanto círculo de estudios redes de aprendizaje y acompañamiento pedagógico en un antes y después de la aplicación de instrumentos. De un $100 \%$ él $70 \%$ de los docentes encuestados del distrito 12D03 consideraron la variable fortalecimiento pedagógico en un nivel medio, el $23 \%$ nivel como bajo y el $7 \%$ nivel alto.

En cuanto a la variable círculos de estudios redes de aprendizaje él $19 \%$ de los docentes encuestados del distrito 12D03 consideraron la dimensión equipo de trabajo en un nivel alto, el $77 \%$ en el nivel medio, un $4 \%$ en el nivel bajo. Especificando que el 
acompañamiento pedagógico si influye significativamente y de evaluación de los resultados.

El presente artículo se ha fundamentado en teorías y trabajos previos y datos estadísticos procesados y analizados donde se midió la incidencia de los círculos de estudios redes de aprendizaje en los fortalecimientos pedagógicos a los docentes a través de la aplicación de un instrumento, análisis y constatación de cada dimensión y el criterio manifestado de los docentes encuestados determinando que, si existe influencia del acompañamiento pedagógico,

En conformidad se determinaron niveles en la variable independiente fortalecimiento pedagógico mediante sus dimensiones y objetivo general. Determinar la incidencia de círculos de estudios redes de aprendizaje en el fortalecimiento pedagógico a docentes en el distrito 12d03 Mocache - Quevedo, Ecuador, 2021. Se demuestra en la tabla 04 de un $100 \%$ en la (encuesta) de la dimensión planificación se encuentra en el nivel medio con el $70 \%$, nivel bajo con el $23 \%$ y el $7 \%$ en nivel alto a referencia a la incidencia de la variable círculos de estudios donde se evidencia en la tabla 5 los encuestados seleccionaron el nivel alto con un $19 \%$ el nivel medio con un $77 \%$ el nivel bajo con un $4 \%$ demostrando que los porcentajes de más altos representa niveles medios, estos resultados los podemos contrastar con lo que dice Tua (2020), quien concluye que se hace necesario potenciar integralmente a los docentes desde la reflexión en lo que los saberes, prácticas y procedimientos así mismo el presente trabajo en concordante la teoría del constructivismo donde se señala que la enseñanza se logra mediante la apropiación adecuada de técnicas e instrumentos y recursos logrando engranajes validos de quienes se educa Peredo (2019).

De la misma forma se consideró lo que dice Jerome Brunner con su teoría sobre el descubrimiento, estimulación e interacción donde el docente es la guía y por eso debe tener predisposición en actualizar sus contenidos continuamente y mejorar su formación y presentar nuevas prácticas educativas que rompan estructuras o barreras que no permiten aplicar nuevas estrategias de aprendizajes (Saborío, 2019).

Con respecto al primer objetivo específico. Determinar la incidencia de círculos de estudios redes de aprendizaje en la planificación del fortalecimiento pedagógico docente, distrito 12d03 Mocache - Quevedo, Ecuador, 2021. 
Se evidencia en la tabla 04 de un $100 \%$ en la (encuesta) se presenta que un $12 \%$ se encuentra en un nivel medio, $58 \%$ en un nivel bajo y $30 \%$ se lo considero con nivel alto confirmando según los resultados son bajos los niveles de planificación a diferencia de lo que manifiesta la dimensión trabajo en equipo perteneciente a la variable circulo de estudios donde en la tabla 5, la figura 2 donde el nivel alto tiene un porcentaje de $19 \%$ y el nivel bajo un $44 \%$.

Información que se puede cotejar con lo que propuso el MINEDUC (2017). Entidad que impulso el fortalecimiento pedagógico a través de la planificación y ejecución de la estrategia círculos de estudios como intercambio de conocimientos dirigido a los docentes de todo el país. En este miso contexto Bettelheim y Charles (2016).Considera que una buena planificación proyecta un buen resultado para cualquier situación en la que se la utilice.

En cuanto al objetivo segundo especifico. Demostrar que los círculos de estudios redes de aprendizaje tienen incidencia en la organización del fortalecimiento pedagógico docente en el distrito 12d03 Mocache - Quevedo, Ecuador, 2021. En cuanto a la dimensión organización en la tabla 04 se muestra del 70 \% corresponde a nivel medio, siendo el porcentaje más alto en comparación con la variable círculos de estudios dimensión interacción social los porcentajes nivel alto con el $26 \%$, nivel medio con un $30 \%$ y el nivel bajo con un $44 \%$. Porcentajes que podemos acotejar con lo que dice Padilla (2013). Que la organización es parte esencial y favorece las capacitaciones que reciben los docentes ya que permite reflexión y logro de los objetivos. En consecuencia Kino (2019). Revela según los resultados de su estudio revela que es preciso organizar capacitaciones que fortalezcan las capacidades de los docentes.

En referencia al tercer objetivo específico Demostrar que los círculos de estudios redes de aprendizajes tienen incidencia en la evaluación del fortalecimiento pedagógico docente en el distrito 12d03 Mocache - Quevedo, Ecuador, 2021.en relación con la dimensión evaluación en la tabla 04 de un $100 \%$ en la (encuesta) se obtuvo el $33 \%$ en nivel medio, y con un nivel bajo un $40 \%$ y apenas con el $27 \%$ en nivel alto.

En comparación con la variable círculos de estudios dimensión interacción social sonde en los resultados del instrumento aplicado los porcentajes se registraron de la siguiente forma en el nivel alto con un $36 \%$ en el nivel medio $31 \%$ y el nivel bajo con un $53 \%$. Ratificando que la evaluación y monitoreo es necesario para cumplir con las metas 
propuestas que mejore las falencias encontradas. Se puede comprobar con el trabajo de Luza (2016). Donde propone un programa de capacitación para docentes aseverando que las capacidades de los docente mejorar con la evaluación y monitoreo continuo.

\section{CONCLUSIÓN O CONSIDERACIONES FINALES}

Se determinó que los círculos de estudio redes de aprendizaje inciden deficientemente en el fortalecimiento pedagógico de los docentes del distrito 12D03, ya que los resultados se presentan de la siguiente manera nivel medio y bajo con el 93\%; diferencia muy significativa con el nivel alto donde solo se obtuvo un $7 \%$ se puede interferir que los porcentajes que se asimilan a los resultados de la variable dependiente círculos de estudios donde el nivel alto se registró con un $19 \%$ el nivel medio con un $77 \%$ el nivel bajo con un $4 \%$ demostrando que los porcentajes de más altos representan al nivel bajo concluyendo que se hace evidente mejorar el programa y reevaluar las capacitaciones que reciben los docentes.

Se determinó que los círculos de estudios redes de aprendizaje no tienen incidencia en la planificación del fortalecimiento pedagógico docente en el distrito 12d03, ya que en los porcentajes de calificación se obtuvo un $12 \%$ en el nivel medio y el nivel alto con un $30 \%$, y el nivel bajo con un $58 \%$ resultados que se pueden corroborar con los datos arrojados en la aplicación del instrumento en la dimensión trabajo en equipo perteneciente donde el nivel alto obtuvo un porcentaje de $19 \%$ y el nivel bajo un $44 \% 19 \%$ y el nivel bajo un $44 \%$. La planificación del programa de capacitación debe ser evaluada y monitoreada continuamente.

Se determinó que los círculos de estudios redes de aprendizajes no tienen incidencia en la organización del fortalecimiento pedagógico docente en el distrito 12d03. En cuanto a los resultados obtenidos de la dimensión evaluación se alcanzó entre los niveles medio y bajo un porcentaje de $73 \%$ a diferencia del nivel alto que obtuvo un $27 \%$ en consecuencia se puede contrastar o interferir estos resultados con los obtenidos en la dimensión interacción social donde el nivel alto con el $26 \%$, nivel medio con un $30 \%$ y el nivel bajo con un $44 \%$. Concluyendo organización en cada una de las capacitaciones deben ser revisada y reformulada.

Se determinó que los círculos de estudios redes de aprendizajes existe muy poca incidencia referente a la evaluación del fortalecimiento pedagógico docente en relación con la dimensión evaluación donde los porcentajes más altos corresponden a los niveles 
bajos con un $93 \%$, y el porcentaje alto con un $63 \%$ resultado se pueden corroborar con los resultados obtenidos de las dos dimensiones evaluación e interacción social concluyendo que es necesario proponer mejoras en la capacitación de los docentes aseverando que las capacidades de los docente mejorar con la evaluación y monitoreo continuo.

\section{LISTA DE REFERENCIAS}

Cantillo (2018). Acompañamiento pedagógico: estrategia para la práctica reflexiva en los docentes de tercer grado de básica primaria. Universidad de la costa Barranquilla. Recuperado de: $\quad$ https://practicareflexiva.pro/wpcontent/uploads/2019/03/Investigacio\%CC\%81n-sobreacompan\%CC\%83amiento-pedagogico.pdf.

Fernández (2017). Apuntes y opiniones sobre educación humanista. Articulo web. Revista entrevista académica Reea. México. Recuperado de: https://www.eumed.net/rev/reea/agosto-18/educacion-humanista.pdf.

Delgado, P (2020). La capacitación docente el gran reto de la educación en línea. Articulo web. Tecnológico de Monterrey, México. Recuperado de: https://observatorio.tec.mx/edu-news/capacitacion-docente-covid.

Gomero (2020). Plan de monitoreo integral pedagógico en el desempeño del docente de Educación Básica Regular. Ancash, 2019. Tesis doctoral. Perú, Chimbote. Recuperado de: https://repositorio.ucv.edu.pe/bitstream/handle/20.500.12692/44389/Gomero_G NI\%20-\%20SD.pdf?sequence=1\&isAllowed=y.

Guber (2001).La Etnografía Método, Campo y Reflexividad. Grupo Editorial Norma Buenos Aires, Barcelona, Caracas, Guatemala, Lima, México, Panamá, Quito, San José, San Juan, San Salvador, Bogotá, Santiag.recuperado de: https://facultad.pucp.edu.pe/comunicaciones/ciudadycomunicacion/wpcontent/uploads/2014/11/Guber_Rosana_La_Etnografia_Metodo_Campo_y_R eflexividad.pdf

Ministerio de Educación del Perú (2015). Guía para la formulación del Plan de Monitoreo (Local / Regional). Recuperado de: http://www.minedu.gob.pe/campanias/pdf/gestion/guia-para-la-formulacion-delplan-de-monitoreo.pdf. 
Ministerio de educación de Ecuador MINEDUC. (2020). Normativa para regular la implementación de la educación abierta en el sistema nacional de educación en tiempos de emergencia. ACUERDO Nro. MINEDUC-MINEDUC-2020-00038A. Quito-Ecuador. Recuperado de: https://educacion.gob.ec/normativa-pararegular-la-implementacion-de-la-educacion-abierta/

Pinto (2018). La Educación Inicial en Bogotá-Colombia y su fundamentación en la Psicología: -La Experiencia de un Jardín Infantil, Tesis Doctoral. Colombia, Manizales. Recuperado De: https://repository.cinde.org.co/bitstream/handle/20.500.11907/2295/TESIS\%20 MAGDALENA.PINTO\%20\%282\%29.pdf?sequence=1\&isAllowed=y.

Ordoñez Ochoa Espinoza (2020). El constructivismo y su prevalencia en el proceso de enseñanza - aprendizaje en la educación básica en Machala. Caso de estudio. Universidad Técnica de Machala. Ecuador. Recuperado de: file:///C:/Users/MINEDUC/Downloads/305-1077-2-PB\%20(1).pdf. 